\title{
LIFE ON EARTH - AN ACCIDENT? Chiral Symmetry and the Anthropic Principle
}

\author{
Ulf-G. MEIßNER* [for the NLEFT Collaboration] \\ HISKP and BCTP, Bonn University, D-53115 Bonn, Germany \\ IAS, IKP and JCHP, Forschungszentrum Jülich D-52425 Jülich, Germany \\ *E-mail: meissner@hiskp.uni-bonn.de \\ www.itkp.uni-bonn.de/ meissner
}

I discuss the fine-tuning of the nuclear forces and in the formation of nuclei in the production of the elements in the Big Bang and in stars.

\section{Definition of the problem}

The elements that are pertinent to life on Earth are generated in the Big Bang and in stars through the fusion of protons, neutrons and nuclei. In Big Bang nucleosynthesis (BBN), alpha particles ( ${ }^{4} \mathrm{He}$ nuclei) and some heavier elements are generated. Life essential elements like ${ }^{12} \mathrm{C}$ and ${ }^{16} \mathrm{O}$ are generated in hot, old stars, where the so-called triple-alpha reaction plays an important role. Here, two alphas fuse to produce the instable, but long-lived ${ }^{8} \mathrm{Be}$ nucleus. As the density of ${ }^{4} \mathrm{He}$ nuclei in such stars is high, a third alpha fuses with this nucleus before it decays. However, to generate a sufficient amount of ${ }^{12} \mathrm{C}$ and ${ }^{16} \mathrm{O}$, an excited state in ${ }^{12} \mathrm{C}$ at an excitation energy of $7.65 \mathrm{MeV}$ with spin zero and positive parity is required as pointed out by Hoyle long ago. ${ }^{1}$ In a further step, carbon is turned into oxygen without such a resonant condition. So we are faced with a multitude of fine-tunings which need to be explained. We know that all strongly interacting composites like hadrons and nuclei must emerge from the underlying gauge theory of the strong interactions, Quantum Chromodynamics (QCD), that is formulated in terms of quarks and gluons. These fundamental matter and force fields are, however, confined. Further, the mass of the light quarks relevant for nuclear physics is very small and thus plays little role in the total mass of nucleons and nuclei. Finally, protons and neutrons form nuclei. This requires the inclusion of electromagnetism, characterized by the fine-structure constant $\alpha_{\mathrm{EM}} \simeq 1 / 137$. So the question we want to 


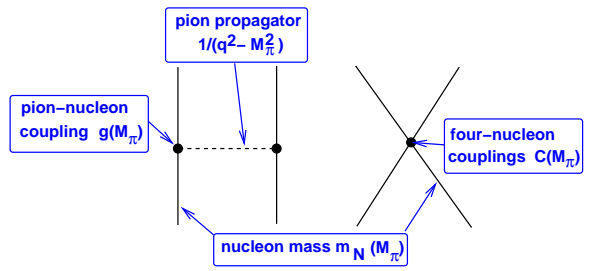

Fig. 1. Explicit and implicit pion (quark) mass dependence of the leading order nucleonnucleon (NN) potential. Solid (dashed) lines denote nucleons (pions).

address in the following is: How sensitive are these strongly interacting composites to variations in the fundamental parameters of QCD+QED? or stated differently: how accidental is life on Earth?

\section{The nuclear force at varying quark mass}

Nuclear forces are best described by utilizing chiral effective field theory (EFT) as pioneered by Weinberg. ${ }^{2}$ The forces between two, three and four nucleons are given by pion-exchange contributions and short-distance multi-nucleon operators, the latter being accompanied by low-energy constants that must be determined by a fit to data. For a review, see Ref. ${ }^{3}$. In this scheme, the quark mass dependence of the forces is generated explicitely (pion propagator) and implicitly (pion-nucleon coupling, nucleon mass, 4N couplings), see Fig.1. Throughout, we use the Gell-MannOakes-Renner relation, $M_{\pi}^{2} \sim\left(m_{u}+m_{d}\right)$, so one can use pion and quark mass dependence synonymously. For any observable $\mathcal{O}$ of a hadron $H$, we can define its quark mass dependence in terms of the so-called $K$-factor, $\delta \mathcal{O}_{H} / \delta m_{f} \equiv K_{H}^{f}\left(\mathcal{O}_{H} / m_{f}\right)$, with $f=u, d, s$, and $m_{f}$ the corresponding quark mass. The pion mass dependence of pion and nucleon properties can be obtained from lattice QCD combined with chiral perturbation theory as detailed in Ref. ${ }^{4}$. The pertinent results are: $K_{M_{\pi}}^{q}=0.494_{-0.013}^{+0.009}$, $K_{F_{\pi}}^{q}=0.048 \pm 0.012$, and $K_{m_{N}}^{q}=0.048_{-0.006}^{+0.002}$, where $q$ denotes the average light quark mass. For the quark mass dependence of the short-distance terms, one has to resort to modeling using resonance saturation. ${ }^{5}$ This induces a sizeable uncertainty that might be overcome by lattice simulations in the future. For the NN scattering lengths, this leads to $K_{1 S 0}^{q}=2.3_{-1.8}^{+1.9}$, $K_{3 S 1}^{q}=0.32_{-0.18}^{+0.17}$ and $K_{\mathrm{BE} \text { (deut) }}^{q}=-0.86_{-0.50}^{+0.45}$ (with BE denoting the binding energy), extending and improving earlier work based on EFTs and models. ${ }^{6-10}$ The running of the NN scattering lengths and the deuteron BE with the light quark mass is shown in Fig. 2. In addition to shifts in $m_{q}$, 

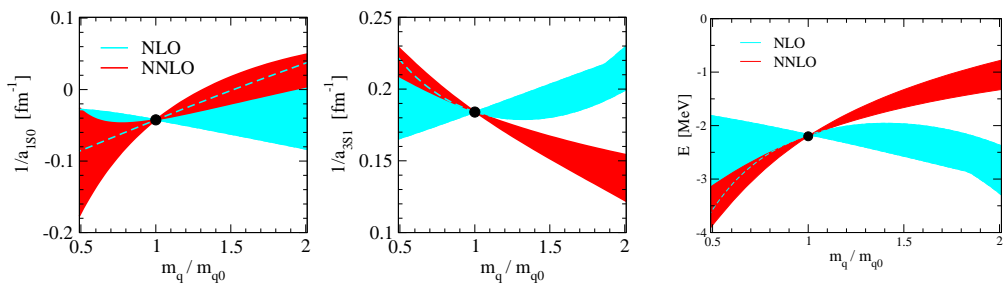

Fig. 2. Quark mass dependence of the inverse scattering length $1 / a_{1 S 0}$ and $1 / a_{3 S 1}$ and the deuteron binding energy. Here, $m_{q 0}$ denotes the physical light quark mass.

we shall also consider the effects of shifts in $\alpha_{\mathrm{EM}}$. The treatment of the Coulomb interaction in the nuclear lattice EFT framework is described in detail in Ref. ${ }^{11}$

\section{Constraints from Big Bang Nucleosynthesis}

With the results from the previous section, one can now analyze what constraints the element abundances in BBN on possible quark mass variations imply. To answer this question, we also need the variation of ${ }^{3} \mathrm{He}$ and ${ }^{4} \mathrm{He}$ with the pion mass. Following Ref. ${ }^{12}$ (BLP), these can be obtained by convoluting the $2 \mathrm{~N} K$-factors with the variation of the 3 - and 4-particle BEs with respect to the singlet and triplet NN scattering lengths. This gives $K_{3_{\mathrm{He}}}^{q}=-0.94 \pm 0.75$ and $K_{3 \mathrm{He}}^{q}=-0.55 \pm 0.42,{ }^{4}$ which is consistent with a direct calculation using nuclear lattice simulations, $K_{3 \mathrm{He}}^{q}=-0.19 \pm 0.25$ and $K_{3 \mathrm{He}}^{q}=-0.16 \pm 0.26 .{ }^{13}$ With this input, we can calculate the BBN response matrix of the primordial abundances $Y_{a}$ at fixed baryon-to-photon ratio, $\delta Y_{a} / \delta m_{q}=\sum_{X_{i}}\left(\delta \ln Y_{a} / \delta \ln X_{i}\right) K_{X_{i}}^{q}$, with $X_{i}$ the relevant BEs for ${ }^{2} \mathrm{H},{ }^{3} \mathrm{H},{ }^{3} \mathrm{He},{ }^{4} \mathrm{He},{ }^{6} \mathrm{Li},{ }^{7} \mathrm{Li}$ and ${ }^{7} \mathrm{BE}$ and the singlet NN scattering length, using the updated Kawano code (for details, see Ref. ${ }^{14}$ ). Combining the calculated with the observed abundances, one finds that the most stringent limits arise from the deuteron abundance $[\mathrm{deut} / \mathrm{H}]$ and the ${ }^{4} \mathrm{He}$ abundance normalized to the one of protons, ${ }^{4} \mathrm{He}\left(Y_{p}\right)$, as most neutrons end up in the alpha nucleus. Combining these leads to the constraint $\delta m_{q} / m_{q}=(2 \pm 4) \%$. In contrast to most earlier determinations, we provide reliable error estimates due to the underlying EFT. However, as pointed out by BLP, one can obtain an even stronger bound due to the neutron lifetime, which strongly affects ${ }^{4} \mathrm{He}\left(Y_{p}\right)$. We have re-evaluated this constraint under the modelindependent assumption that all quark and lepton masses vary with the Higgs vacuum expectation value $v$, leading to

$$
|\delta v / v|=\left|\delta m_{q} / m_{q}\right| \leq 0.9 \% .
$$




\section{The fate of carbon-based life as a function of the fundamental parameters of QCD+QED}

I now turn to the central topic of this talk, namely how fine-tuned is the production of carbon and oxygen with respect to changes in the fundamental parameters of QCD+QED? Or, stated differently, how much can we detune these parameters from their physical values to still have an habitable Earth as shown in Fig. 3. To be more precise, we must specify which parameters we can vary. In QCD, the strong coupling constant is tied to the nucleon mass through dimensional transmutation. However, the light quark mass (here, only the strong isospin limit is relevant) is an external parameter. Naively, one could argue that due to the small contribution of the quark masses to the proton and the neutron mass, one could allow for sizeable variations. However, the relevant scale to be compared to here is the average binding energy per nucleon, $E / A \leq 8 \mathrm{MeV}$ (which is much smaller than the nucleon mass). As noted before, the Coulomb repulsion

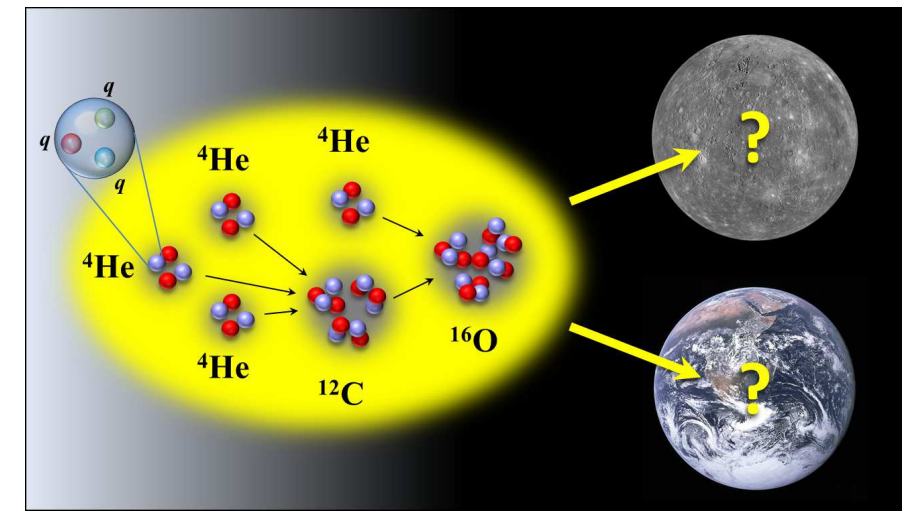

Fig. 3. Graphical representation of the question of how fine-tuned is life on Earth under variations of the average light quark mass and $\alpha_{\mathrm{EM}}$. Figure courtesy of Dean Lee.

between protons is an important ingredient in nuclear binding, therefore we must also consider changes in $\alpha_{\mathrm{EM}}$. The tool to do this are nuclear lattice simulations, which allowed e.g. for the first $a b$ initio calculation of the Hoyle state. ${ }^{15}$ Let us consider first QCD (for details, see Refs. ${ }^{16,17}$ ). We want to calculate the variations of the pertinent energy differences in the triple-alpha process $\delta \Delta E / \delta M_{\pi}$, which according to Fig. 1 boils down to (we 
consider small variations around the physical value of the pion mass $\left.M_{\pi}^{\mathrm{ph}}\right)$ :

$$
\begin{aligned}
\left.\frac{\partial E_{i}}{\partial M_{\pi}}\right|_{M_{\pi}^{\mathrm{ph}}} & =\left.\frac{\partial E_{i}}{\partial \tilde{M}_{\pi}}\right|_{M_{\pi}^{\mathrm{ph}}}+\left.x_{1} \frac{\partial E_{i}}{\partial m_{N}}\right|_{m_{N}^{\mathrm{ph}}}+\left.x_{2} \frac{\partial E_{i}}{\partial \tilde{g}_{\pi N}}\right|_{\tilde{g}_{\pi N}^{\mathrm{ph}}} \\
& +\left.x_{3} \frac{\partial E_{i}}{\partial C_{0}}\right|_{C_{0}^{\mathrm{ph}}}+\left.x_{4} \frac{\partial E_{i}}{\partial C_{I}}\right|_{C_{I}^{\mathrm{ph}}},
\end{aligned}
$$

with the definitions

$$
\left.x_{1} \equiv \frac{\partial m_{N}}{\partial M_{\pi}}\right|_{M_{\pi}^{\mathrm{ph}}},\left.\left.\quad x_{2} \equiv \frac{\partial g_{\pi N}}{\partial M_{\pi}}\right|_{M_{\pi}^{\mathrm{ph}}} x_{3} \equiv \frac{\partial C_{0}}{\partial M_{\pi}}\right|_{M_{\pi}^{\mathrm{ph}}},\left.\quad x_{4} \equiv \frac{\partial C_{I}}{\partial M_{\pi}}\right|_{M_{\pi}^{\mathrm{ph}}},
$$

with $\tilde{M}_{\pi}$ the pion mass appearing in the pion-exchange potentials The various derivatives in Eq. (2) can be obtained precisely using Auxiliary Field Quantum Monte Carlo techniques and the $x_{i}(i=1,2,3,4)$ are related to the pion and nucleon $K$-factors determined in Sec. 2 . The schemedependent quantities $x_{3,4}$ can be traded for the pion-mass dependence of the inverse singlet and triplet scattering lengths, $\bar{A}_{s} \equiv \partial a_{s}^{-1} /\left.\partial M_{\pi}\right|_{M_{\pi}^{\mathrm{ph}}}$, $\bar{A}_{t} \equiv \partial a_{t}^{-1} /\left.\partial M_{\pi}\right|_{M_{\pi}^{\mathrm{ph}}}$. We can then express all energy differences appearing in the triple-alpha process $\left(\Delta E_{b} \equiv E_{8}-2 E_{4}, \Delta E_{h} \equiv E_{12}^{\star}-E_{8}-E_{4}, \varepsilon=\right.$ $E_{12}^{\star}-3 E_{4}$, with $E_{4}$ and $E_{8}$ for the energies of the ground states of ${ }^{4} \mathrm{He}$ and ${ }^{8} \mathrm{Be}$, respectively, and $E_{12}^{\star}$ denotes the energy of the Hoyle state) as functions of $\bar{A}_{s}$ and $\bar{A}_{t}$. One finds that all these energy differences are correlated, i.e. the various fine-tunings in the triple-alpha process are not independent of each others, see the left panel of Fig. 4. Further, one finds a strong dependence on the variations of the ${ }^{4} \mathrm{He} \mathrm{BE}$, which is strongly suggestive of the $\alpha$-cluster structure of the ${ }^{8} \mathrm{Be},{ }^{12} \mathrm{C}$ and Hoyle states. Such correlations related to the production of carbon have indeed been speculated upon earlier. ${ }^{18,19}$ Consider now the reaction rate of the triple-alpha process as given by $r_{3 \alpha} \sim N_{\alpha}^{3} \Gamma_{\gamma} \exp \left(-\varepsilon / k_{\mathrm{B}} T\right)$, with $N_{\alpha}$ the $\alpha$-particle number density in the stellar plasma with temperature $T, \Gamma_{\gamma}=3.7(5) \mathrm{meV}$ the radiative width of the Hoyle state and $k_{B}$ is Boltzmann's constant. The stellar modeling calculations of Refs. ${ }^{20,21}$ suggest that sufficient abundances of both carbon and oxygen can be maintained within an envelope of $\pm 100 \mathrm{keV}$ around the empirical value of $\varepsilon=379.47(18) \mathrm{keV}$. This condition can be turned into a constraint on shifts in $m_{q}$ that reads (for more details, see Ref. ${ }^{17}$ )

$$
\left|\left[0.572(19) \bar{A}_{s}+0.933(15) \bar{A}_{t}-0.064(6)\right]\left(\frac{\delta m_{q}}{m_{q}}\right)\right|<0.15 \% \text {. }
$$

The resulting constraints on the values of $\bar{A}_{s}$ and $\bar{A}_{t}$ compatible with the condition $|\delta \varepsilon|<100 \mathrm{keV}$ are visualized in the right panel of Fig. 4. The 

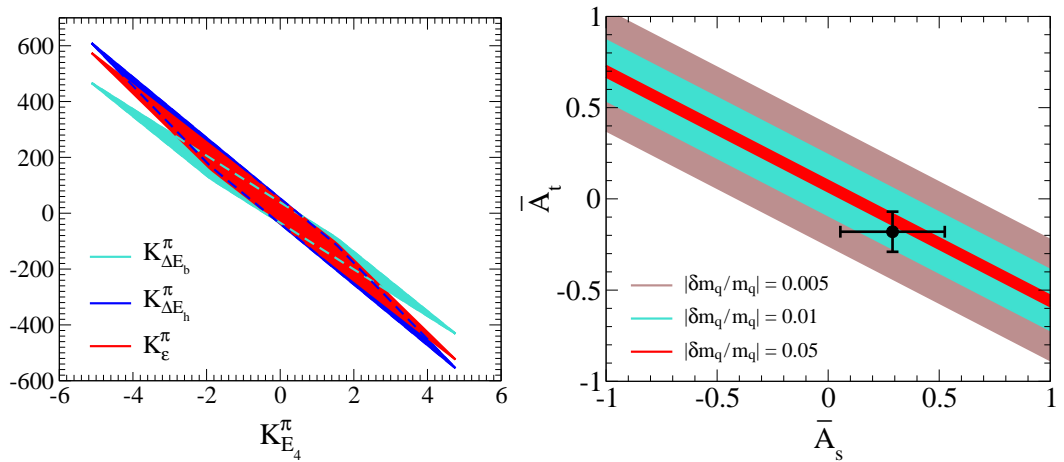

Fig. 4. Left panel: Sensitivities of $\Delta E_{h}, \Delta E_{b}$ and $\varepsilon$ to changes in $M_{\pi}$, as a function of $K_{E_{4}}^{\pi}$ under independent variation of $\bar{A}_{s}$ and $\bar{A}_{t}$ over the range $\{-1 \ldots 1\}$. The bands correspond to $\Delta E_{b}, \varepsilon$ and $\Delta E_{h}$ in clockwise order. Right panel: "Survivability bands" for carbon-oxygen based life from Eq. (4), due to $0.5 \%$ (broad outer band), $1 \%$ (medium band) and $5 \%$ (narrow inner band) changes in $m_{q}$ in terms of the input parameters $\bar{A}_{s}$ and $\bar{A}_{t}$. The most up-to-date $\mathrm{N}^{2} \mathrm{LO}$ analysis of $\bar{A}_{s}$ and $\bar{A}_{t}$ from Ref. ${ }^{14}$ is given by the data point with horizontal and vertical error bars.

various shaded bands in this figure cover the values of $\bar{A}_{s}$ and $\bar{A}_{t}$ consistent with carbon-oxygen based life, when $m_{q}$ is varied by $0.5 \%, 1 \%$ and $5 \%$. Given the current theoretical uncertainty in $\bar{A}_{s}$ and $\bar{A}_{t}$, our results remain compatible with a vanishing $\partial \varepsilon / \partial M_{\pi}$, in other words with a complete lack of fine-tuning. Interestingly, Fig. 4 (right panel) also indicates that the triplealpha process is unlikely to be fine-tuned to a higher degree than $\simeq 0.8 \%$ under variation of $m_{q}$. The central values of $\bar{A}_{s}$ and $\bar{A}_{t}$ from Ref. ${ }^{14}$ suggest that variations in the light quark masses of up to $2-3 \%$ are unlikely to be catastrophic to the formation of life-essential carbon and oxygen. A similar calculation of the tolerance for shifts in the fine-structure constant $\alpha_{\mathrm{EM}}$ suggests that carbon-oxygen based life can withstand shifts of $\simeq 2.5 \%$ in $\alpha_{\mathrm{EM}} \cdot$

\section{A short discussion of the anthropic principle}

The Hoyle state dramatically increases the reaction rate of the triple-alpha process. The resulting enhancement is also sensitive to the exact value of $\varepsilon$, which is therefore the principal control parameter of this reaction. As the Hoyle state is crucial to the formation of elements essential to life as we know it, this state has been nicknamed the "level of life" ${ }^{22}$ Thus, the Hoyle state is often viewed as a prime manifestation of the anthropic principle, which states that the observable values of the fundamental physical and 
cosmological parameters are restricted by the requirement that life can form to determine them, and that the Universe be old enough for that to occur. ${ }^{23,24}$ See, however, Ref. ${ }^{25}$ for a thorough historical discussion of the Hoyle state in view of the anthropic principle. We remark that in the context of cosmology and string theory, the anthropic principle and its consequences have had a significant influence, as reviewed recently in. ${ }^{26}$ As noted already in Ref., ${ }^{19}$ the allowed variations in $\varepsilon$ are not that small, as $|\delta \varepsilon / \varepsilon| \simeq 25 \%$ still allows for carbon-oxygen based life. So one might argue that the anthropic principle is indeed not needed to explain the fine-tunings in the triple-alpha process. However, as we just showed, this translates into allowed quark mass variations of $2-3 \%$ and modifications of the fine-structure constant of about $2.5 \%$. The fine-tuning in the fundamental parameters is thus much more severe than the one in the energy difference $\varepsilon$. Therefore, beyond such relatively small changes in the fundamental parameters, the anthropic principle indeed appears necessary to explain the observed abundances of ${ }^{12} \mathrm{C}$ and ${ }^{16} \mathrm{O}$.

\section{Summary and outlook}

In this talk, I have summarized recent developments in our understanding of the fine-tuning in the generation of the life-essential elements as well as the light elements generated in $\mathrm{BBN}$. As shown, the allowed parameter variations in QCD+QED are small, giving some credit to the anthropic principle. To sharpen these conclusions, future work is required. On one side, lattice QCD at sufficiently small quark masses will eventually be able to give tighter constraints on the parameters $\bar{A}_{s, t}$ and on the other side, nuclear lattice simulations have to be made more precise to reduce the theoretical error in the binding and excitation energies and to provide $a b$ initio calculations of nuclear reactions, for first steps, see Refs. ${ }^{27,28}$.

\section{Acknowledgments}

I would like to thank my NLEFT collaborators Evgeny Epelbaum, Hermann Krebs, Timo Lähde and Dean Lee for a most enjoyable collaboration. I also thank the organizers for their perfect job. Work supported in part by DFG and NSFC (Sino-German CRC 110), Helmholtz Association (contract VH-VI-417), BMBF (grant 05P12PDFTE), and the EU (HadronPhysics3 project) Computational resources provided by the Jülich Supercomputing Centre (JSC) at the Forschungszentrum Jülich and by RWTH Aachen. 


\section{References}

1. F. Hoyle, Astrophys. J. Suppl. Ser. 1, 121 (1954).

2. S. Weinberg, Phys. Lett. B 251, 288 (1990).

3. E. Epelbaum, H.-W. Hammer, and U.-G. Meißner, Rev. Mod. Phys. 81, 1773 (2009).

4. J. C. Berengut, E. Epelbaum, V. V. Flambaum, C. Hanhart, U.-G. Meißner, J. Nebreda, and J. R. Peláez, Phys. Rev. D 87, 085018 (2013).

5. E. Epelbaum, U.-G. Meißner, W. Gloeckle and C. Elster, Phys. Rev. C 65, 044001 (2002)

6. H. Müther, C. A. Engelbrecht and G. E. Brown, Nucl. Phys. A 462, 701 (1987).

7. S. R. Beane and M. J. Savage, Nucl. Phys. A 713, 148 (2003).

8. E. Epelbaum, U.-G. Meißner and W. Gloeckle, Nucl. Phys. A 714, 535 (2003).

9. V. V. Flambaum and R. B. Wiringa, Phys. Rev. C 76, 054002 (2007).

10. J. Soto and J. Tarrus, Phys. Rev. C 85, 044001 (2012).

11. E. Epelbaum, H. Krebs, D. Lee, and U.-G. Meißner, Eur. Phys. J. A 45, 335 (2010).

12. P. F. Bedaque, T. Luu, and L. Platter, Phys. Rev. C 83, 045803 (2011).

13. T. Lähde, private communication.

14. J. C. Berengut, V. V. Flambaum and V. F. Dmitriev, Phys. Lett. B 683, 114 (2010).

15. E. Epelbaum, H. Krebs, D. Lee, and U.-G. Meißner, Phys. Rev. Lett. 106, 192501 (2011).

16. E. Epelbaum, H. Krebs, T. A. Lähde, D. Lee and U.-G. Meißner, Phys. Rev. Lett. 110, 112502 (2013).

17. E. Epelbaum, H. Krebs, T. A. Lähde, D. Lee and U.-G. Meißner, Eur. Phys. J. A 49, 82 (2013).

18. M. Livio, D. Hollowell, A. Weiss, and J. W. Truran, Nature 340, 281 (1989).

19. S. Weinberg, "Facing Up" (Harvard University Press, Cambridge, Massachusetts, 2001).

20. H. Schlattl, A. Heger, H. Oberhummer, T. Rauscher, and A. Csótó, Astrophys. Space Sci. 291, 27 (2004).

21. H. Oberhummer, A. Csótó, and H. Schlattl, Nucl. Phys. A 689, 269 (2001).

22. A. Linde, "The inflationary multiverse," in Universe or multiverse?, edited by B. Carr (Cambridge University Press, Cambridge, England, 2007).

23. B. Carter, "Large number coincidences and the anthropic principle", in Confrontation of cosmological theories with observational data, edited by M. S. Longair (Reidel, Dordrecht, 1974).

24. B. J. Carr and M. Rees, Nature 278, 605 (1979).

25. H. Kragh, Arch. Hist. Exact Sci. 64, 721 (2010).

26. A. N. Schellekens, arXiv:1306.5083 [hep-ph].

27. G. Rupak and D. Lee, Phys. Rev. Lett. 111, 032502 (2013).

28. M. Pine, D. Lee and G. Rupak, Eur. Phys. J. A 49, 151 (2013). 